\title{
Eugenol-loaded microspheres incorporated into textile substrates
}

\author{
M. G. Simões - P. Coimbra • A. S. Carreira • M. M. Figueiredo • \\ M. H. Gil · P. N. Simões
}

Received: 5 August 2019/ Accepted: 20 January 2020/Published online: 10 February 2020

(C) The Author(s) 2020

\begin{abstract}
This work reports studies on the production of eugenol-loaded microspheres by solvent evaporation method, targeted for incorporation into textile substrates, using different cellulose derivatives: ethyl cellulose, cellulose acetate (CA), cellulose butyrate acetate, and cellulose acetate phthalate. The microspheres were evaluated in terms of size, shape, thermal stability, encapsulation efficiency, and eugenol kinetics release. CA-based microspheres proved to be the best, thus being selected for subsequent experiments. Comparable properties of the CA-based microspheres were achieved ongoing from $100 \mathrm{~mL}$ to higher batch
\end{abstract}

volumes (up to $2000 \mathrm{~mL}$ ). The eugenol-loaded microspheres were successfully incorporated into cotton fabrics using a padding technique, confirmed by FESEM. Eugenol release profiles from the impregnated textiles demonstrated a slow and controlled release (less than $20 \%$ of the encapsulated amount over 90 days). The developed microspheres demonstrated to be the most promising for the retention and protection of hydrophobic active compounds for possible textile applications.

M. G. Simões $(\bowtie) \cdot$ P. Coimbra · A. S. Carreira · M. M. Figueiredo · M. H. Gil · P. N. Simões CIEPQPF, Department of Chemical Engineering, University of Coimbra, Polo II, Pinhal de Marrocos, 3030-790 Coimbra, Portugal

e-mail: monicasimoes@eq.uc.pt

M. G. Simões

Institute of Bioproducts and Paper Technology, Graz University of Technology, Inffeldgasse 23/EG, 8010 Graz, Austria

A. S. Carreira

Devan - Micropolis, S.A., Moreira da Maia, Portugal 


\section{Graphic abstract}

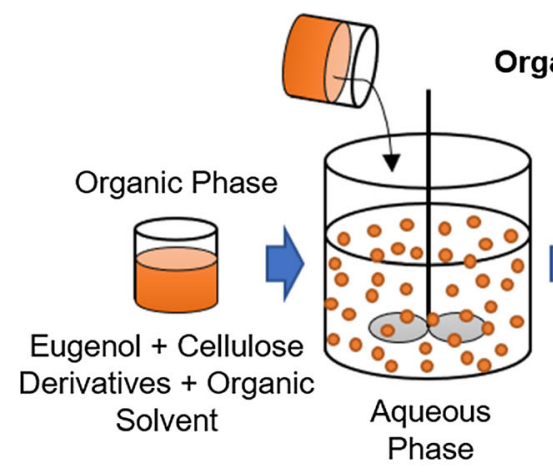

Textile Functionalization - Padding Technique
Organic Solvent Evaporation

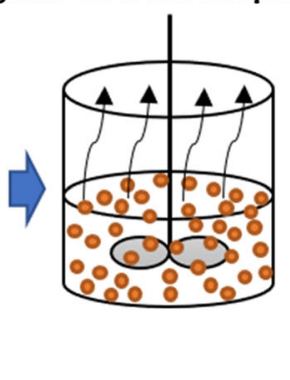

Eugenol loaded Microspheres

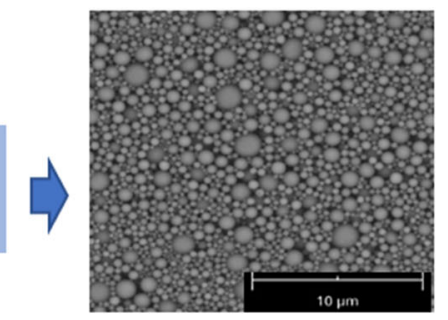

\section{Cotton Fabric functionalized with Eugenol} loaded Microspheres

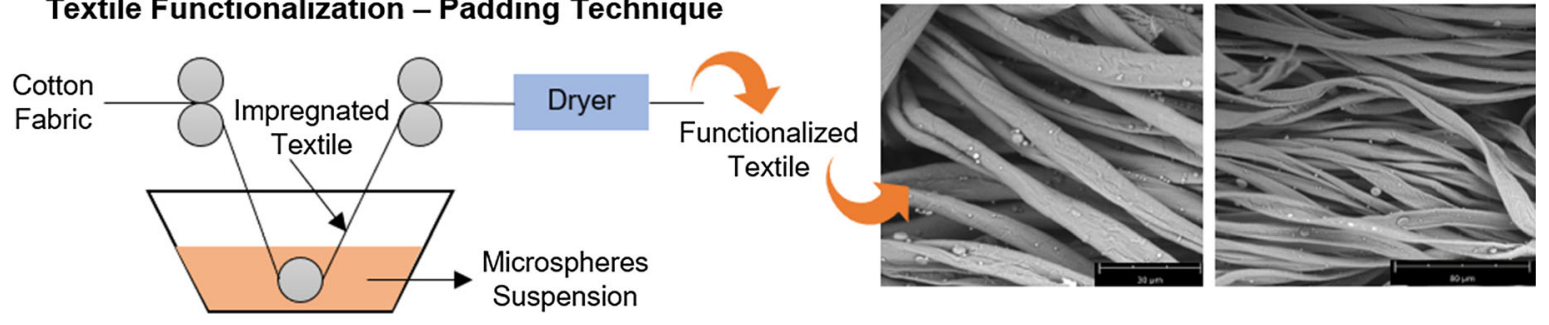

Keywords Microencapsulation - Cellulose derivatives · Eugenol $\cdot$ Control release $\cdot$ Textile functionalization

\section{Introduction}

The textile industry has undergone significant reforms over the last years because of scientific and technological progress. The major changes are related to the demands of consumers looking for materials with greater comfort and new functionalities but also concerned about environmental issues (Sarier and Onder 2007). Textile companies are currently interested in developing unique, environmentally friendly and high-quality products, namely "smart textiles" that improve safety, aesthetics, health, and well-being (Prasad 2007; Jamekhorshid et al. 2014; Salaün 2016). These functionalities are normally achieved by the incorporation of active compounds which, in most cases, cannot be added to the textile substrates using traditional technologies. In fact, it is often necessary to make use of strategies capable of protecting the active agents from evaporation or from oxidation/deactivation reactions with the surrounding environment. Microencapsulation followed by subsequent incorporation into textile substrates is a common approach (Carfagna and Persico 2006) that overcomes many of the aforementioned problems, and additionally can result in an efficient control delivery system (Ghosh 2006; Sarier and Onder 2007). Besides, microencapsulation processes for textile functionalization are significantly encouraged since they give the possibility of producing a high volume of suitable microparticles at low cost (Nelson 2002). The microencapsulation of phase change materials (Jamekhorshid et al. 2014), and essential oils (Sutaphanit and Chitprasert 2014; Patil et al. 2016), are the most successful examples of encapsulated active materials for textile functionalization (Petrulis and Petrulyte 2019).

Among the most selected coating materials for the microparticles, polymers occupy a relevant place (Campos et al. 2013a). However, due to serious concerns about environmental issues related to nonbiodegradable materials, the use of non-biodegradable polymers for the production of short-life products is not recommended (Avérous and Pollet 2012). As a consequence, there is a generalized effort to replace these environmentally harmful compounds by biodegradable polymers (Chandra and Rustgi 1998; 
Kumari et al. 2010; Martins et al. 2014). Macromolecules derived from biomass, notably polysaccharides, represent a very important class of biodegradable polymers due to their abundance, accessibility, biocompatibility, biological activity, and the easy processability into various forms, such as films, particles, fibers and capsules (Campos et al. 2013b). Among the polysaccharides, cellulose and its derivatives and chitosan are worth mentioning.

In the present work, aiming to understand the potential of different cellulose derivatives for the microencapsulation of eugenol, cellulose acetate butyrate $(\mathrm{CAB})$, ethyl cellulose (EC), cellulose acetate (CA) and cellulose acetate phthalate (CAP) were selected and tested, at small scale $(100 \mathrm{~mL}$ batch volumes), as encapsulants (Doelker 1993; Miyazaki et al. 2003; Fundueanu et al. 2005; Jelvehgari and Montazam 2012; Graham et al. 2014; Pan-in et al. 2014).

Eugenol, the active agent to encapsulate, is the main compound of clove oil with strong antiseptic, antibacterial and antiviral properties (Cortés-rojas et al. 2014; Piletti et al. 2017). Eugenol has also been reported to be successfully retained using other biodegradable polymers, like gelatin, alginate and chitosan (Shinde and Nagarsenker 2011; Woranuch and Yoksan 2013; Shao et al. 2018).

The solvent evaporation method was the selected microencapsulation technique due to its simplicity, the capacity of encapsulation of hydrophobic compounds like eugenol, as well as due to its ability to produce microspheres suitable for textile applications (Coimbra et al. 2008).

The cellulose derivative and surfactant concentration, eugenol/cellulose derivative ratio, and organic phase/aqueous phase ratio used were the same for all cellulose derivatives, and the produced microspheres were evaluated in terms of size and size distribution, shape, thermal stability, encapsulation efficiency, and eugenol release profiles. After preliminary experiments, the most promising cellulose derivative was used for higher microsphere production volumes (up to $2000 \mathrm{~mL}$ ).

The microspheres can be incorporated into the textile substrates using traditional finishing processes, such as coating, spraying and exhaustion or using impregnation by padding. These processes allow adding new functionalities to the fabrics, like appearance, texture, and breathability without changing their original features. In this work, the fibers were impregnated with the microspheres by padding using a foulard followed by a drying step (Ghosh 2006; Rodrigues et al. 2009). To evaluate the incorporation of eugenol-loaded microspheres into the fabrics, the textile substrates were analyzed by FE-SEM. Finally, the eugenol release profile of the textile-microsphere conjugate was assessed.

\section{Materials and methods}

Materials

Eugenol (99\%, Acrõs Organics, Belgium), poly(vinyl alcohol) (PVA, Mw 115,000, VWR Chemicals, USA), cellulose acetate butyrate (CAB, Mw 16,000, butyryl content 50-54\%, Acrõs Organics, USA), ethyl cellulose (EC, ethoxyl content 48\%, 300 cps, Acrõs Organics, USA), cellulose acetate (CA, Mw 100,000, Acrõs Organics, USA), cellulose acetate phthalate (CAP) (Mw 2500, Sigma Aldrich, USA), ethyl acetate (VWR Chemicals, France), chloroform (VWR Chemicals, France), ethanol (VWR Chemicals, France) and fabric composed of $100 \%$ cotton (knit, weight of $150 \mathrm{~g} / \mathrm{m}^{2}$, from Devan Chemicals, Belgium).

Methods

\section{Cellulose derivatives microsphere preparation}

Eugenol-loaded microspheres were prepared by an oilin-water $(\mathrm{O} / \mathrm{W})$ emulsion solvent evaporation method (Fig. 1), based on the procedure described by Coimbra et al. (Coimbra et al. 2008) in $100 \mathrm{~mL}$ batch volumes.

To form the $\mathrm{O} / \mathrm{W}$ emulsion, the organic phase, containing Eugenol and the cellulose derivative, was added dropwise to the aqueous phase (PVA solution) and left under homogenization for $10 \mathrm{~min}$ at $8000 \mathrm{rpm}$ with an Ultraturrax T25 (IKA, Germany). For all tests, the cellulose derivative and surfactant concentration were $3 \%(\mathrm{w} / \mathrm{v})$ and $0.5 \%(\mathrm{w} / \mathrm{v})$, respectively. A $1: 1(\mathrm{w} /$ w) eugenol/cellulose derivative ratio and 1:5 (v/v) organic phase/aqueous phase ratio were used. The cellulose derivatives $\mathrm{CA}$ and $\mathrm{CAB}$ were dissolved in ethyl acetate whereas the derivatives EC and CAP were dissolved in a 50/50 (v/v) mixture of Chloroform/Ethanol. The obtained $\mathrm{O} / \mathrm{W}$ emulsions were continuously stirred for $12 \mathrm{~h}$, to allow the total 


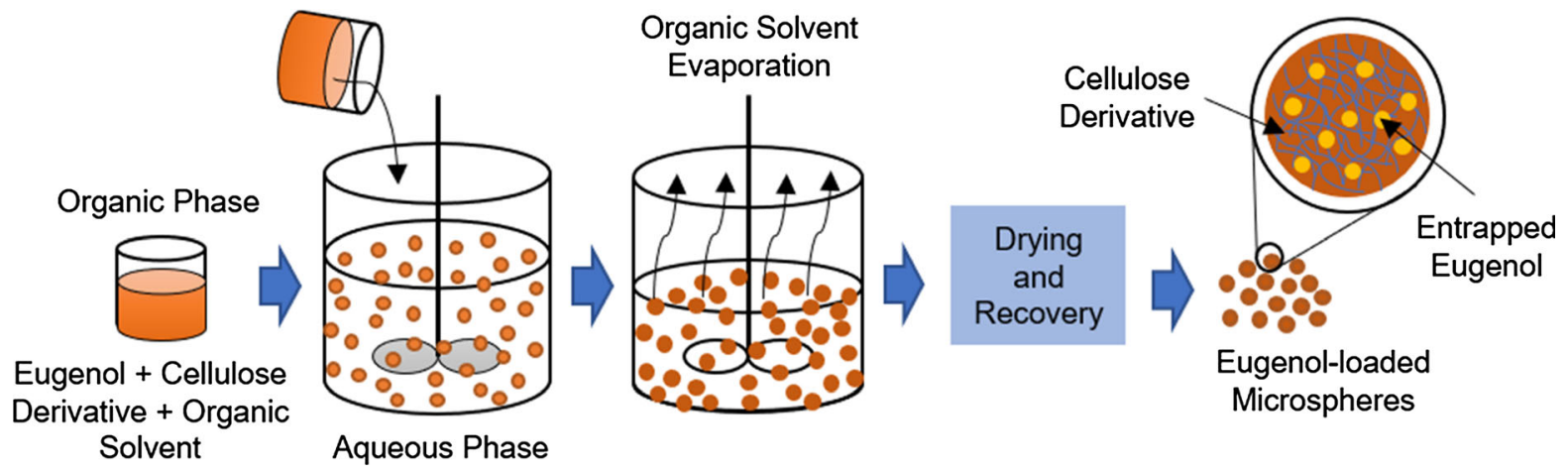

Fig. 1 Microencapsulation of eugenol with cellulose derivatives by the emulsion solvent evaporation method

evaporation of the organic solvent. The resulting microspheres were collected by centrifugation, at $3000 \mathrm{rpm}$, and then washed three times with deionized water. To prevent eugenol losses, the samples were frozen at $-18{ }^{\circ} \mathrm{C}$ and freeze-dried in an Alpha 1-2 LD Plus (CHRIST, Germany), operating at $-52{ }^{\circ} \mathrm{C}$ in a pressure chamber of 0.04 mbar. The dried microspheres were stored in a desiccator until further use.

\section{Larger scale CA microsphere production}

In these experiments, carried out in total volumes of 250, 500, 1000 and $2000 \mathrm{~mL}$, only cellulose acetate (CA) was used as the coating material for the microencapsulation of eugenol. To increase the mass yield of the microspheres, the CA concentration in the organic phase was increased from $3 \%$ (used in $100 \mathrm{~mL}$ tests, section "Cellulose derivatives microsphere preparation") to $7 \%(\mathrm{w} / \mathrm{v})$ in ethyl acetate and added dropwise to the aqueous phase, PVA $0.5 \%(\mathrm{w} / \mathrm{v})$ solution to form the O/W emulsion, at the same conditions of the $100 \mathrm{~mL}$ scale assays (section "Cellulose derivatives microsphere preparation"). Eugenol/cellulose derivative and organic/aqueous phase ratios (specified in section "Cellulose derivatives microsphere preparation") were also kept invariant. Due to the higher volume of $\mathrm{O} / \mathrm{W}$ emulsions in the larger scale tests, a rotary evaporator $\left(40{ }^{\circ} \mathrm{C}\right.$, 0.1 mbar, Rotavapor ${ }^{\circledR}$ B-114, Buchi, Switzerland) was used to promote total evaporation of the organic solvent of the $\mathrm{O} / \mathrm{W}$ emulsion. The produced microspheres were collected by centrifugation, washed, freeze-dried and stored in a desiccator.

\section{Size distribution}

Particle size distributions of the microspheres were measured by static light scattering using a Mastersizer 2000E (Malvern Instruments Ltd., United Kingdom) optical bench fitted with a Hydro 2000MU sample dispersion unit. For this analysis, sonicated samples were added to $800 \mathrm{~mL}$ beaker of distilled water $(\mathrm{pH}$ 6.5) to achieve an adequate light obscuration (between $7 \%$ and 10\%) and pumped at $2000 \mathrm{rpm}$. For the dispersed phase and the continuous phase, refractive index values of 1.57 and 1.33 , respectively, were used. $\mathrm{d} 10$, d50, and d90 values represent the diameter for which a given percentage (10, 50 and $90 \%$, respectively) of particles is smaller than that size. Measurements were repeated, on average, ten times per sample.

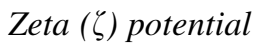

$\zeta$-potential measurements were performed in a Zetasizer Nano-Z (Malvern Instruments, Malvern, United Kingdom) using a combination of measurement techniques: Electrophoresis and Laser Doppler Velocimetry (Laser Doppler Electrophoresis). The $\zeta$ potential values were provided directly by the instrument. In all measurements, microspheres were dispersed in distilled water $(\mathrm{pH} 6.5)$ at $25^{\circ} \mathrm{C}$. $\zeta$-potential was obtained as the average of 10 readings per sample.

\section{Thermogravimetric analysis (TGA)}

The thermal stability of the produced microspheres was assessed by High-Resolution Thermogravimetry Analysis (Hi-Res-TGA). A TA Instruments Q500 
thermogravimetric analyzer (thermobalance sensitivity: $0.1 \mu \mathrm{g}$ ) was used after being calibrated over the temperature range $25-500{ }^{\circ} \mathrm{C}$ by measuring the Curie point of nickel standard in open platinum crucibles, under a dry nitrogen purge flow of $100 \mathrm{~mL} \mathrm{~min}{ }^{-1}$, and a (maximum) heating rate of $2{ }^{\circ} \mathrm{C} \mathrm{min}^{-1}$. At least two measurements were performed for each sample.

\section{Encapsulation efficiency and release profiles}

UV spectrophotometry was used to determine the encapsulation efficiency (EE) and release profiles of eugenol (Pramod et al. 2013). To determine the eugenol content in the microspheres, a known amount of lyophilized eugenol-loaded microspheres was dispersed in ethanol $\left(1 \mathrm{mg} \mathrm{mL}^{-1}\right)$ and kept in these conditions for $48 \mathrm{~h}$, under moderate agitation, to promote the total release and dissolution of the entrapped eugenol. The resulting suspension was then centrifuged, and the supernatant was filtrated and analyzed in a UV spectrophotometer (Jasco, model V550 , USA) at $280 \mathrm{~nm}$. The encapsulation efficiency $\mathrm{EE}(\%)$ is defined as the ratio between the experimentally determined eugenol content (EEL) and the theoretical eugenol loadings (TEL):

$$
E E=\frac{E E L}{T E L}
$$

where

$$
E E L=\frac{\text { weight of detected eugenol }}{\text { weight of eugenol loaded microspheres }}
$$

and

$$
T E L=\frac{\text { weight of added eugenol }}{\text { weight of added eugenol }+ \text { weight of added polymer }}
$$

The "weight of detected eugenol" is the amount of eugenol quantified by UV spectrophotometry and the "weight of eugenol loaded microspheres" the weight of eugenol microspheres in ethanol used for the measurement.

For the release profiles, the remaining lyophilized eugenol-loaded microsphere samples from the same batch (weighted and left exposed at room atmosphere) were periodically collected and treated as mentioned above to obtain the absorbance measurements that enable the calculation of the amount of eugenol that endured in the microspheres and thus the eugenol released over time. All curves were normalized for analysis.

\section{Textile functionalization}

A $100 \%$ cotton fabric with a weight of $150 \mathrm{~g} \mathrm{~m}^{-2}$ was impregnated with the eugenol-loaded microspheres (Fig. 2). This textile was treated using a finishing formulation with a microsphere concentration of $35 \mathrm{~g} \mathrm{~L}^{-1}$ leading to a wet pick-up of $70 \%$. Microspheres were applied by padding using a horizontal foulard mangle of pneumatic type (RAPID foulard, model P-B0, from Labortex CO., Ltd) at room temperature and pressure of 4 bar. The impregnated textile was then dried at $80{ }^{\circ} \mathrm{C}$ for $10 \mathrm{~min}$.

For the release profiles, the textile substrates functionalized with the eugenol-loaded microspheres were cut in ca. $4 \mathrm{~cm}^{2}$ samples and left exposed at room atmosphere for 90 days to allow the eugenol release. Textile samples were periodically collected and dipped in $6 \mathrm{~mL}$ of ethanol for $48 \mathrm{~h}$ to promote the total dissolution of the entrapped eugenol. The final solutions of dissolved eugenol in ethanol were then used in the absorbance measurements as previously described (section "Encapsulation efficiency and release profiles") to calculate the eugenol release over time. All curves were normalized to compare the eugenol release between the lyophilized microspheres and the microspheres entrapped in the textile.

\section{Surface morphology}

Particle shape and surface morphologies of the microspheres were analyzed by field emission scanning electron microscopy (FE-SEM). Microspheres were placed on double-sided graphite tape attached to a metal surface without any coating. Observations were performed at $1.5 \mathrm{kV}$ in a MERLIN GEMIN II (Zeiss, Germany).

\section{Results and discussion}

Smaller scale microsphere production

For the functionalization of textile substrates, the microparticles must be stable, biodegradable, exhibit a good retention ability (EE), and a controlled release capability, which in this case means a moderate 


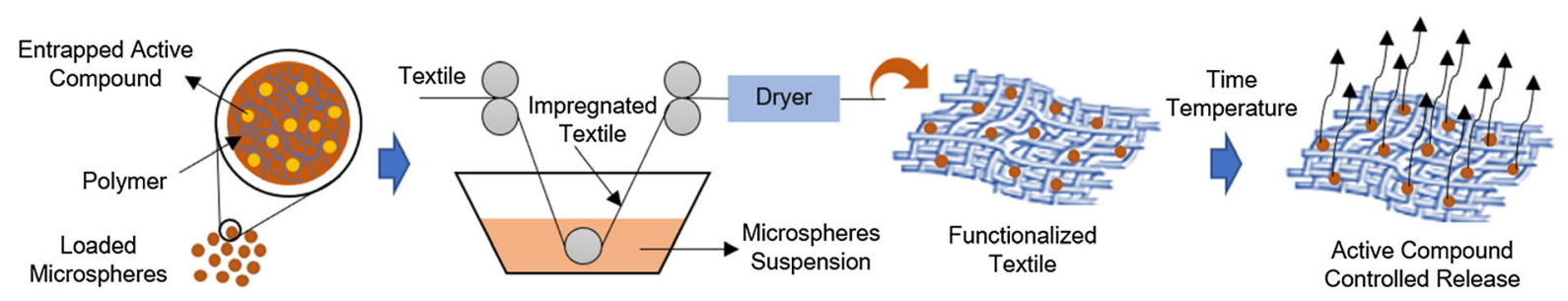

Fig. 2 Production of a functionalized textile with controlled release capacity microspheres. The microspheres contain an active compound of interest entrapped in the polymeric matrix

release of the encapsulated content over a $2 / 3$-month interval. Considering that the microparticles will be housed between the fibers of the fabric, too large size particles are not desirable to prevent their early loss (e.g., due to friction). Ideally, sizes below $15 \mu \mathrm{m}$ are desirable. For this application, the polydispersity and morphology of the microparticles are not critical factors, but uniform size distributions and a spherical shape contribute to their stability.

As mentioned, different cellulose derivatives (CA, $\mathrm{CAB}, \mathrm{CAP}$, and $\mathrm{EC}$ ) were used to produce microspheres at a small scale with the necessary characteristics to microencapsulate eugenol employing an oilin-water method. These cellulose derivatives were used in a concentration of $3 \%(\mathrm{~m} / \mathrm{v})$ in the solvents described in section "Cellulose derivatives microsphere preparation" (ethyl acetate for $\mathrm{CA}$ and $\mathrm{CAB}$ derivatives and a mixture of Chloroform/Ethanol for EC and CAP). The produced microspheres were primarily analyzed in terms of size distribution and morphology.

Size distribution curves reveal considerable differences depending on the cellulose derivatives used. As evidenced by the results from Fig. 3a, CA and $\mathrm{CAB}$ microspheres are much smaller than the EC and CAP microspheres (the largest particle sizes of the former two correspond approximately to the lowest sizes of the latter samples). Moreover, the size distribution of CA exhibits a slightly asymmetric unimodal distribution, with a peak at $1.3 \mu \mathrm{m}$ (Table 1 ; top), and a small tail towards larger particles, whereas the CAB microspheres have a bimodal distribution, with a prominent peak at $2.2 \mu \mathrm{m}$ and a smaller peak at $1.3 \mu \mathrm{m}$ (Table 1; top). Besides, the particle size distributions of EC and CAP based microspheres, ranging from 4 to $35 \mu \mathrm{m}$, exhibit peaks at $11.5 \mu \mathrm{m}$ and $20 \mu \mathrm{m}$, respectively (Table 1; top).

These findings are in line with FE-SEM images (Fig. 4) which clearly show that $\mathrm{CA}$ and $\mathrm{CAB}$
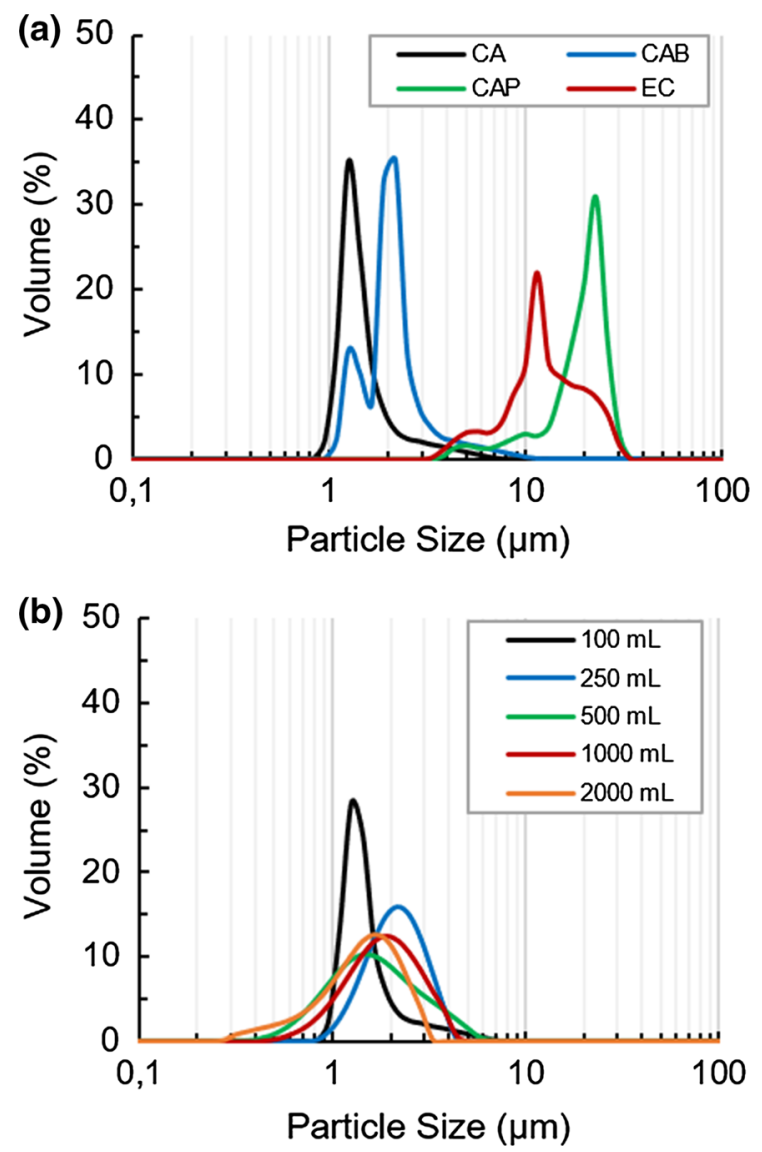

Fig. 3 Size distributions curves of the eugenol-loaded microspheres at a smaller and $\mathbf{b}$ larger scales

microspheres (Fig. 4a, b) are much smaller than those of the two other samples. Additionally, CA-based microspheres are slightly smaller than those prepared using CAB. Nonetheless, they both seem fairly homogeneous in size and shape. On the other hand, Fig. $4 \mathrm{c}, \mathrm{d}$ allow to inferring the presence of much larger particles, with a broader size range, in the case of EC and CAP. All samples exhibit spherical particles with a smooth surface, except EC-based particles 
Table 1 Size, surface charge, and efficiency of encapsulation $(\mathrm{EE})$ of the eugenol-loaded $\mathrm{CA}, \mathrm{CAB}$, $\mathrm{CAP}$ and EC microspheres produced at different scales (the tabulated results correspond to mean values \pm standard deviation, with $\mathrm{n}=3$ for particle sizes and EE and $\mathrm{n}=10$ for zeta-potential)

\begin{tabular}{|c|c|c|c|c|c|c|}
\hline Sample & $d_{10} / \mu \mathrm{m}$ & $d_{50} / \mu \mathrm{m}$ & $d_{90} / \mu \mathrm{m}$ & Mode/ $\mu \mathrm{m}$ & $\zeta$-potential/mV & $\mathrm{EE} / \%$ \\
\hline \multicolumn{7}{|c|}{ Smaller Scale (100 mL) Production (all samples) } \\
\hline $\mathrm{CA}$ & $0.54 \pm 0.02$ & $1.21 \pm 0.01$ & $4.87 \pm 0.02$ & 1.26 & $-28.3 \pm 1.2$ & $34.7 \pm 0.1$ \\
\hline $\mathrm{CAB}$ & $0.98 \pm 0.01$ & $2.85 \pm 0.01$ & $7.22 \pm 0.02$ & 2.19 & $-28.7 \pm 0.6$ & $24.6 \pm 0.2$ \\
\hline CAP & $8.27 \pm 1.26$ & $19.62 \pm 0.69$ & $29.89 \pm 3.81$ & 19.95 & $-29.5 \pm 0.9$ & $31.4 \pm 0.1$ \\
\hline EC & $2.65 \pm 0.44$ & $11.73 \pm 0.44$ & $28.54 \pm 1.38$ & 11.48 & $-31.8 \pm 0.6$ & $18.5 \pm 0.3$ \\
\hline$V_{\mathrm{Batch} / \mathrm{mL}}$ & $d_{10} / \mu \mathrm{m}$ & $d_{50} / \mu \mathrm{m}$ & $d_{90} / \mu \mathrm{m}$ & Mode/ $\mu \mathrm{m}$ & $\zeta$-potential/mV & $\mathrm{EE} / \%$ \\
\hline \multicolumn{7}{|c|}{ Larger Scale Production (CA) } \\
\hline 250 & $1.28 \pm 0.39$ & $2.00 \pm 0.50$ & $2.99 \pm 0.64$ & 2.18 & $-22.9 \pm 3.8$ & $39.8 \pm 0.2$ \\
\hline 500 & $0.78 \pm 0.01$ & $1.50 \pm 0.50$ & $3.12 \pm 0.90$ & 1.44 & $-19.4 \pm 0.4$ & $36.8 \pm 0.1$ \\
\hline 1000 & $0.95 \pm 0.13$ & $1.73 \pm 0.03$ & $2.91 \pm 0.04$ & 1.91 & $-18.9 \pm 0.2$ & $34.5 \pm 0.1$ \\
\hline 2000 & $0.63 \pm 0.04$ & $1.37 \pm 0.02$ & $2.22 \pm 0.02$ & 1.66 & $-18.3 \pm 0.4$ & $33.3 \pm 0.1$ \\
\hline
\end{tabular}
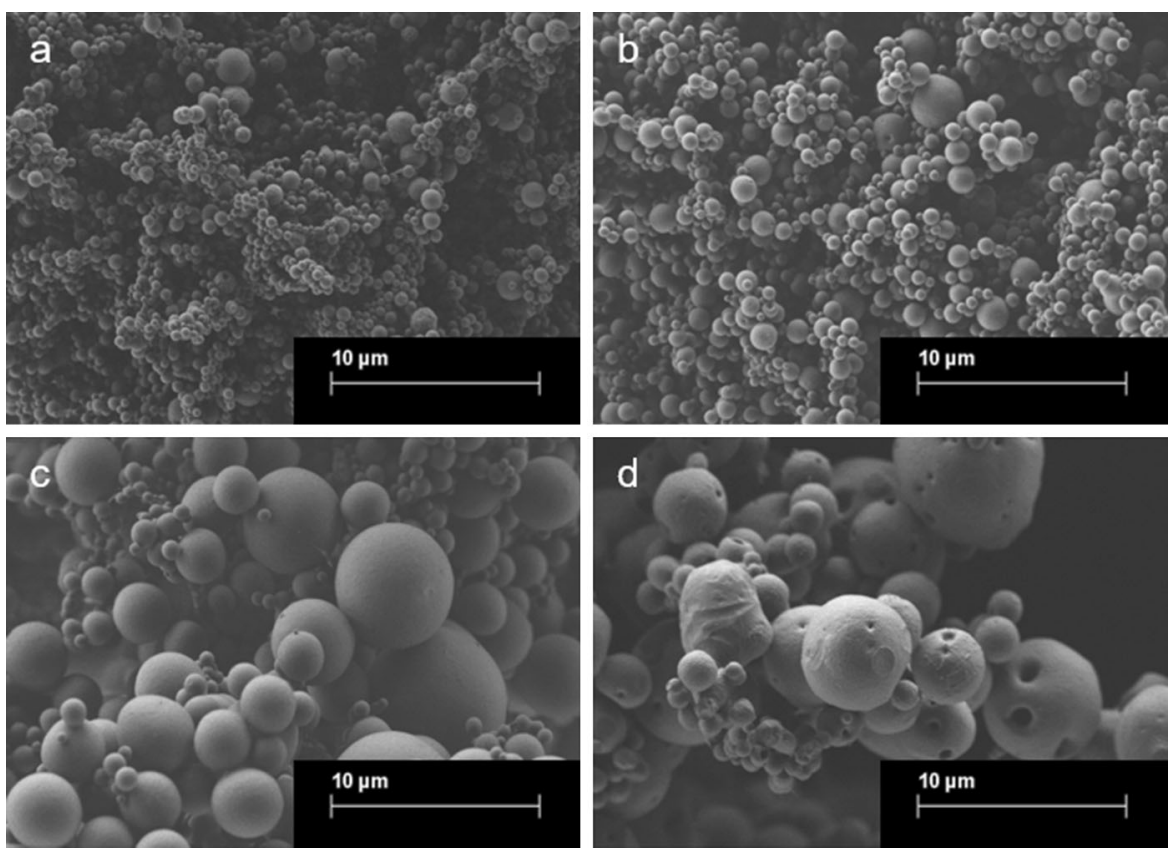

Fig. 4 FE-SEM images of the eugenol-loaded CA (a), CAB (b), CAP (c) and EC (d) microspheres produced at smaller scale (magnification of $1.50 \mathrm{~K}$ ) which exhibit surface irregularities (suggesting pores and/or holes), as well as less sphericity (Fig. 4d). It is worth mentioning that for the FE-SEM analysis lyophilized microspheres were used, and therefore the samples consisted of a powder whose tendency to agglomerate is visible in the SEM images (Fig. 4). However, this should be understood as a side effect caused by the lyophilization process.

The obtained results (Fig. 3a, Table 1; top and Fig. 4) demonstrated that the set formed by CA and CAB-based microspheres presents smaller sizes
$(<10 \mu \mathrm{m})$ and relatively narrow size distributions. On the other hand, the set including EC and CAPbased microspheres exhibits larger particles (up to $35 \mu \mathrm{m})$ and broader size distributions. The noticeable differences between these group of samples can be explained by the different solvents used, since the immiscibility of the organic phase in the aqueous phase is crucial for the $\mathrm{O} / \mathrm{W}$ emulsion (Coimbra et al. 2008; Jyothi et al. 2010; Campos et al. 2016). In fact, ethanol is water-miscible; thus, when the organic phase of cellulose derivatives EC and CAP is 
gradually added to the aqueous phase, it is likely that the solvent mixture will partially dissolve in the water. This phenomenon somehow disrupts the emulsion and may lead to the formation of microspheres with a more pronounced polydispersity, i.e. broader size distributions (Fig. 3a, Table 1; top and Fig. 4).

In terms of zeta potential (Table 1; top), all cellulose derivatives led to negatively charged particles, with average values around $-29 \mathrm{mV}$. This result suggests moderate stability of the suspensions, meaning that the propensity to aggregate might not be a critical issue (Torchilin 2006; Kumar and Dixit 2017).

The eugenol encapsulation efficiencies (EE) are rather different between samples: $35 \%$ for CA, $31 \%$ for CAP, $25 \%$ for CAB, and 19\% for EC (Table 1; top). The fact that EC based microparticles are those with the lowest EE value can also be explained by the aforementioned reasons, i.e. since eugenol is highly soluble in ethanol (Pramod et al. 2013) and this is water-soluble, a loss of eugenol can occur when this is added to the aqueous phase. This, however, does not explain the relatively high EE value found for CAP based microspheres $(31 \%)$, that also use this same solvent. Another explanation that can be advanced is related to the interaction/affinity of eugenol with cellulose derivatives. Indeed, the basic structure of the functional groups of cellulose derivatives $\mathrm{CA}, \mathrm{CAB}$ and CAP are similar to each other but quite different from EC. This can also be a relevant factor for eugenol retention.

HiRes-TGA curves and, for a better mass loss rate comparison, their time derivatives, are depicted in Fig. 5 for eugenol, CA, CAB, CAP, and EC, as well as for the resultant microspheres. Table 2 summarizes thermoanalytical quantities of interest. When considered alone both $\mathrm{CA}$ and $\mathrm{CAB}$ exhibit essentially one decomposition stage with peak temperatures $(333.3 \pm 0.1){ }^{\circ} \mathrm{C}$ and $(337.2 \pm 0.2){ }^{\circ} \mathrm{C}$, respectively, whereas CAP decomposes through two consecutive steps (peak temperatures $(195.2 \pm 0.3){ }^{\circ} \mathrm{C}$ and $(331.21 \pm 0.50){ }^{\circ} \mathrm{C}$, respectively), the first ascribable to the elimination of acetyl and phthalyl groups, and the second to the degradation of the backbone chain (Vijayalakshmi Rao et al. 2000). Overall, the thermoanalytical results demonstrate that the cellulose derivatives, both alone and in the form of eugenolloaded microspheres, are thermally stable. Considering that the first mass loss stage observed at ca. $73-78{ }^{\circ} \mathrm{C}$ in the eugenol-loaded microspheres is, in all likelihood, due to the release (vaporization) of eugenol (and not to the decomposition of the polymeric matrix), the actual decomposition temperatures $\left(T_{\text {on }}\right)$ start well above $200{ }^{\circ} \mathrm{C}$ or even $300{ }^{\circ} \mathrm{C}$ in most cases. The thermal stability (considering the main decomposition event) follows the order $\mathrm{CAB}>\mathrm{EC}>$ $\mathrm{CA} \cong \mathrm{CAP}$. The residual mass is lower than ca. $11 \%$ in most samples, being rather small for the ECbased samples. It is worth mentioning that a plausible explanation for the observed differences in the TGA profile on going from the CAP raw polymer to the CAP microspheres with Eugenol rests on the fact that the latter may cause changes in the specific area of the polymeric matrix. Similar thermal behavior has been reported in the study of nanostructured CAP (Olaru et al. 2013), in which different profiles between the thermal decomposition of CAP-based film/fibers and powder were found.

The HiRes-TGA data (Fig. 5 and Table 2) confirm the good thermal stabilities of the prepared microspheres, which is an important attribute for further application (processing). Indeed, this information is crucial for selecting the operating conditions of the textile functionalization with the eugenol-loaded microspheres. The profiles of both TG and DTG curves of eugenol alone clearly indicate a vaporization process. The measured temperatures at weight loss $2 \%$ and $5 \%$ for eugenol were close to 74 and $86{ }^{\circ} \mathrm{C}$, respectively. These data together with the overall $\mathrm{TG}$ (and DTG) profiles in Fig. 5 allow to unequivocally ascribe the first mass loss stage of microspheres to the release of the eugenol they incorporate.

The eugenol release profiles were assessed to study the control release capacity of the cellulose derived microspheres. As can be seen in Fig. 6a, at the same conditions, EC based microspheres are the faster releasers, followed by $\mathrm{CAP}, \mathrm{CAB}$, and $\mathrm{CA}$ microspheres, able to better retain eugenol. The maximum percentage of eugenol release is dependent on the cellulose derivative used in the microencapsulation, varying from $35 \%$ for the CA-based microspheres to $80 \%$ of the EC profile over 63 days, which is a quite interesting result. Comparing the release profiles of Fig. 6a and the encapsulated efficiency values of Table 1 it can be concluded that, although retaining less eugenol, the EC based microspheres exhibit a faster release rate, probably favored by the incorporating pores (Fig. 4d). 

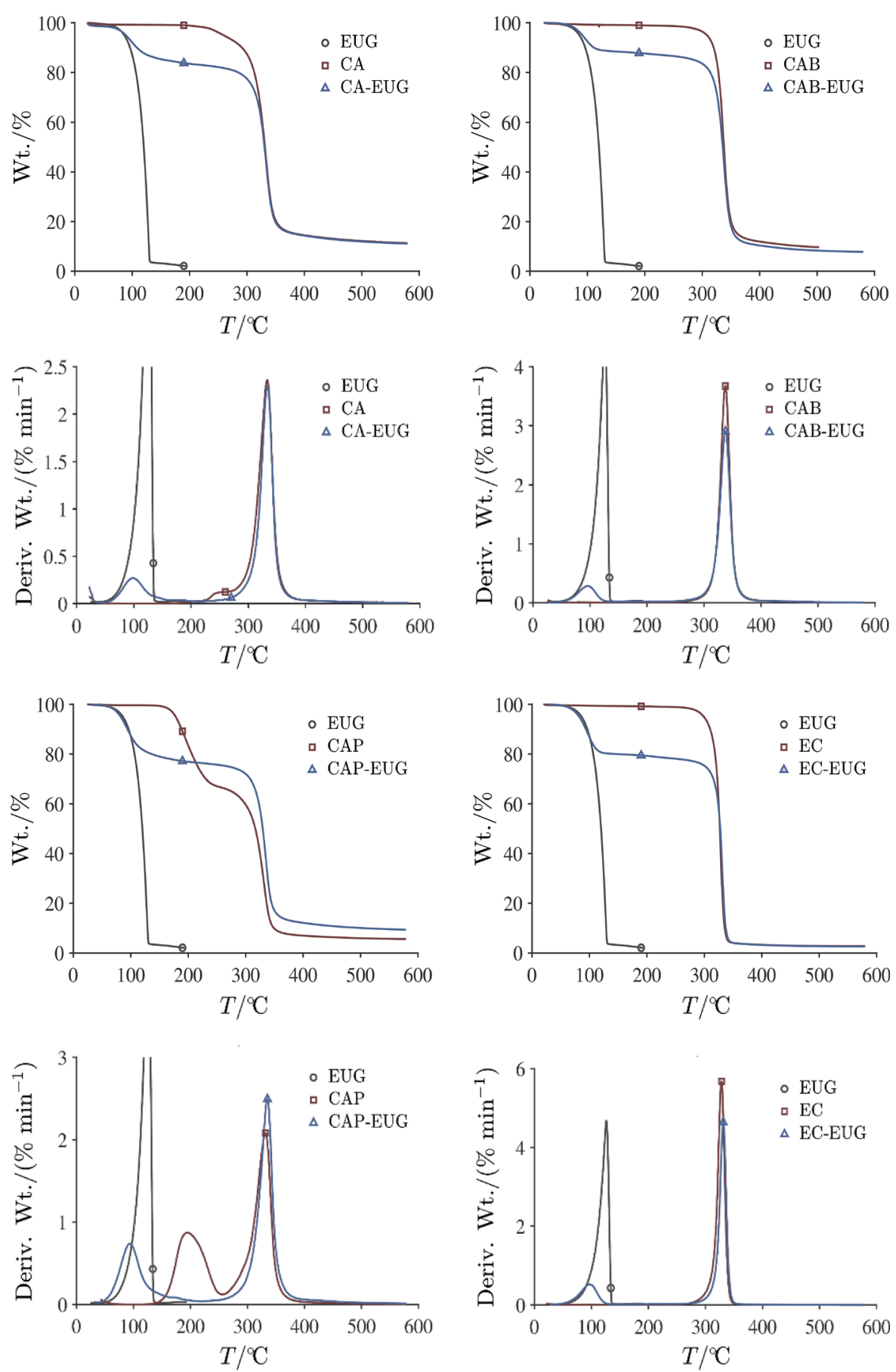

Fig. 5 HiRes-TGA and time derivative (DTG) curves of pure eugenol, CA, CAB, CAP and EC biodegradable cellulose derivatives, and of the corresponding eugenol-loaded microspheres

Characteristics such as particle size, morphology, polydispersity, thermal stability, and, to a less extent, the encapsulation efficiencies and the release profiles, were the criteria taken into consideration for the selection of cellulose derivatives for further studies. CAP and EC-based microspheres were excluded 
Table 2 Characteristic quantities (average and standard deviation of at least two runs) derived from the thermoanalytical curves (cf. Fig. 5)

\begin{tabular}{|c|c|c|c|c|c|c|c|}
\hline Sample & $T_{\text {on }} /{ }^{\circ} \mathrm{C}$ & & $T_{\mathrm{p}} /{ }^{\circ} \mathrm{C}$ & & $\Delta m / \%$ & & $m_{\mathrm{r}} / \%$ \\
\hline CA & $234.9 \pm 1.8$ & $314.3 \pm 0.3$ & $333.3 \pm 0.1$ & & $4.87 \pm 0.64$ & $80.33 \pm 0.78$ & $10.78 \pm 0.69$ \\
\hline CA-EUG & $77.9 \pm 0.3$ & $317.4 \pm 0.9$ & $98.5 \pm 0.5$ & $333.4 \pm 0.1$ & $15.34 \pm 0.10$ & $69.39 \pm 0.30$ & $11.07 \pm 0.01$ \\
\hline CAP & $171.5 \pm 0.5$ & $311.4 \pm 1.2$ & $195.2 \pm 0.3$ & $331.2 \pm 0.5$ & $32.01 \pm 0.94$ & $58.01 \pm 1.63$ & $5.47 \pm 0.00$ \\
\hline CAP-EUG & $73.0 \pm 0.2$ & $319.8 \pm 0.2$ & $93.5 \pm 0.1$ & $335.3 \pm 0.4$ & $23.51 \pm 0.13$ & $63.73 \pm 0.49$ & $9.36 \pm 0.15$ \\
\hline $\mathrm{CAB}$ & $325.6 \pm 1.2$ & & $337.2 \pm 0.2$ & & $87.34 \pm 0.45$ & & $9.61 \pm 0.10$ \\
\hline CAB-EUG & $76.2 \pm 0.2$ & $324.4 \pm 1.3$ & $95.3 \pm 0.4$ & $337.3 \pm 0.4$ & $12.42 \pm 0.06$ & $76.99 \pm 0.33$ & $7.98 \pm 0.22$ \\
\hline $\mathrm{EC}$ & $318.8 \pm 0.4$ & & $328.0 \pm 0.2$ & & $96.7 \pm 0.08$ & & $2.74 \pm 0.06$ \\
\hline EC-EUG & $77.1 \pm 0.2$ & $322.4 \pm 0.1$ & $95.8 \pm 0.3$ & $331.0 \pm 0.3$ & $20.77 \pm 0.01$ & $76.52 \pm 0.64$ & $2.41 \pm 0.21$ \\
\hline
\end{tabular}

$T_{\text {on }}$ : extrapolated onset temperature (TG curve); $T_{\mathrm{p}}$ : peak temperature (DTG curve); $\Delta m$ : mass loss in relevant events (TG curve); $m_{\mathrm{r}}$ : residual mass (TG curve)
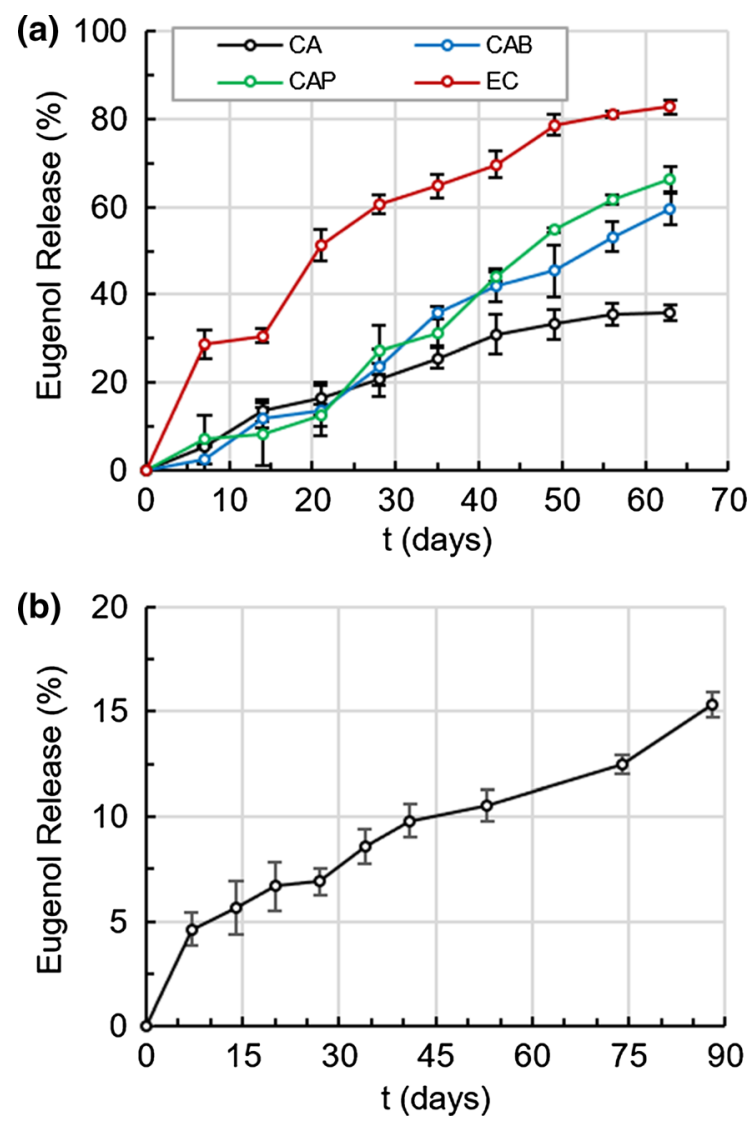

Fig. 6 Eugenol release profiles of a CA, CAB, CAP and EC eugenol-loaded microspheres and $\mathbf{b}$ the functionalized textile with eugenol-loaded CA microspheres produced in a higher production volume (presented results refer to mean average \pm standard deviation, $\mathrm{n}=3$ ) because of their size and release capacity (see Figs. 3, 4, 5, 6 and Table 1; top). Among the two other cellulose derivatives (CA and $\mathrm{CAB}$ ) which presented similar sizes, morphology and polydispersity (Fig. 3a, 4a-e and Table 1; top), CA-based microparticles revealed a higher encapsulation efficiency and a slower eugenol release profile (Table 1; top and Fig. 6a). Summing up, from the analysis of the above-described results, the eugenol CA-based microspheres incorporating eugenol proved to possess not only a uniform size distribution but also the highest encapsulation efficiency, as well as the best-controlled release capacity. Therefore, CA was the cellulose derivative selected for the subsequent larger-scale tests and to a tentative evaluation of the incorporation of CA-based microspheres on textile fibers.

\section{Larger scale CA microsphere production}

To check the effects of larger-scale production, total volumes of $250,500,1000$ and $2000 \mathrm{~mL}$ were tested, maintaining the eugenol/cellulose derivative and organic phase/aqueous phase ratios. Moreover, the percentage of CA in the organic phase was increased from 3 to $7 \%(\mathrm{w} / \mathrm{v})$ to enhance the final mass yield.

Figure $3 \mathrm{~b}$ compares the particle size distribution curves of CA-based microspheres for the batch volumes from 250 to $2000 \mathrm{~mL}$ with the curve corresponding to the smaller scale experiments (100 $\mathrm{mL}$ batch). The most obvious feature is the 
widening of the size distributions and a shift towards larger values when going from the $100 \mathrm{~mL}$ scale to the larger-scale tests. Among the latter, there are no very significant differences in both the overall distributions and the marginal variations in their parameters. (Table 1; bottom and Fig. 3b). The increase in production volume involved an increase in the cellulose derivative concentration (from 3 to $7 \%$ (w/v)) thus increasing the viscosity of the organic phase and hindering its dispersion in the aqueous phase; this explains the occurrence of larger microspheres and broader size distributions.

The FE-SEM image corresponding to the $250 \mathrm{~mL}$ batch (Fig. 7a) indicates that the morphology of the CA-based microspheres was maintained (see also Fig. 4a), i.e. smooth spherical microparticles ranging from ca. 1 to $4 \mu \mathrm{m}$. Thus, the increase in the production volume in the studied range (250-2000 mL) has no noticeable effects on the size, polydispersity, and morphology of the microspheres.

The larger-scale experiments led to lower (absolute) zeta potential values $(-20 \mathrm{mV}$ on average; Table 1; bottom) than the preliminary counterparts (ca. - $28 \mathrm{mV}$, Table 1; bottom). Nevertheless, no aggregates were found in the suspensions during its characterization or later textile impregnation.

The encapsulation efficiencies (Table 1: bottom) are in general slightly higher than those of the smaller scale tests, a result that validates the beneficial effect of increasing the concentration of CA. However, as production volume increases, EE values tend to slightly decrease. Taking into consideration that for larger batch volumes (up to 8 times) the suspension homogenization is getting more difficult, the obtained results are quite satisfactory, confirming the viability of the formulation and the selected technique.
Textile impregnation

An exploratory textile functionalization test was performed by the padding technique (Fig. 2) to evaluate how the selected cellulose derivative-based microspheres behave in the textile matrix in terms of eugenol release performance. No specific physi$\mathrm{cal} / \mathrm{chemical}$ attachment procedure was used in the impregnation process so that eugenol-load microspheres were simply entrapped into the cotton fibers of the textile substrate.

Figure $7 b, c$ present FE-SEM images of the textiles after impregnation with CA microspheres (from a $500 \mathrm{~mL}$ batch). Although sparsely populated, a fairly homogenous distribution of the microspheres throughout the fabric, without significant agglomeration, and preserving the original spherical shape, is observed. This indicates that the particles are resistant to the applied pressure during the padding process. The thermal stability characterizing the CA microspheres as demonstrated by the TGA results ensures its integrity after the drying stage. However, it should be noted that some volatilization of eugenol and concomitant leaking from the microspheres can occur when exposed to $80{ }^{\circ} \mathrm{C}$ for $10 \mathrm{~min}$, indicating that some technical tuning might be necessary regarding the textile functionalization procedure.

The resultant impregnated fabric contained $0.02 \mathrm{~g} /$ $\mathrm{g}$ (wt. of dry microspheres per wt. of fabric), value obtained by the difference between the weight of untreated and treated fabric (Rodrigues et al. 2009); which is clearly low, mainly because, as mentioned, no assembly strategy was applied in the impregnation process, microspheres are only entangled into the textile fibers.
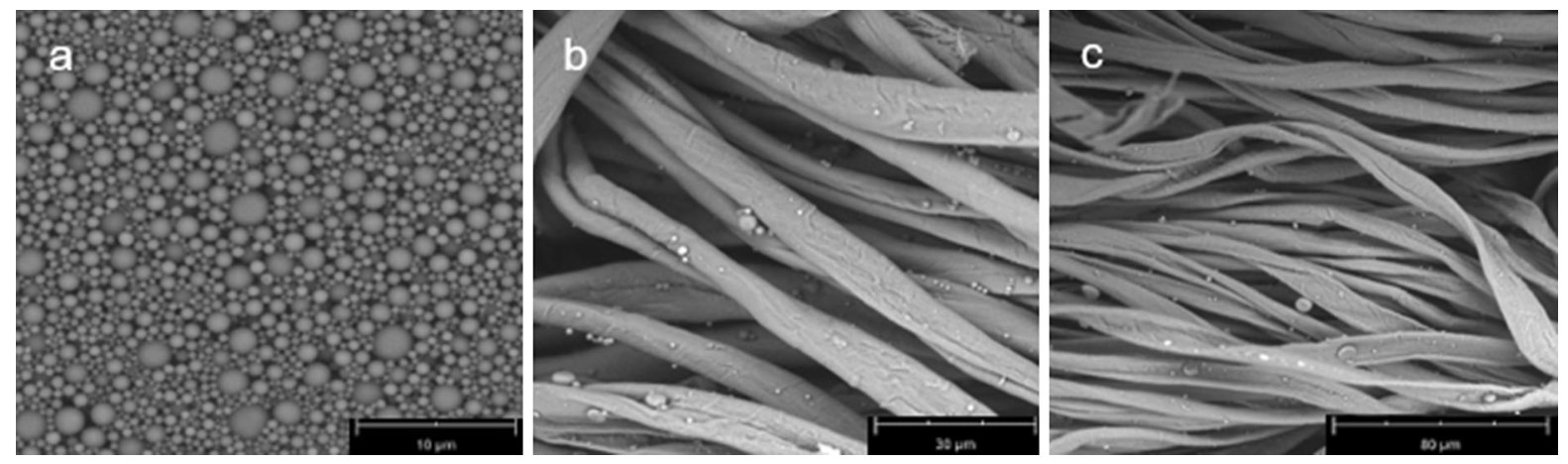

Fig. 7 FE-SEM images of the functionalized textile substrate (b, c) with eugenol-loaded CA microspheres (a) 
Additionally, eugenol release profiles from the microsphere-functionalized textile were recorded (section "Textile functionalization"). These tests were carried out in contact with air in controlled temperature and humidity ambient, at the same conditions used in the release experiments carried out with the lyophilized microspheres alone. The release profile from the textile over 90 days (Fig. 5b) shows that only $15 \%$ of the total initial amount of retained eugenol was released by diffusion in the air. This value is considerably lower than that detected for the release of eugenol from lyophilized microspheres alone (Fig. 6a) that reached about $35 \%$ (of the total initial amount of retained eugenol) after 60 days, indicating that incorporation of the microspheres into the fibers implies longer retention of eugenol.

\section{Conclusions}

The study here reported shows that cellulose acetate was the most adequate cellulose derivative tested for the microencapsulation of eugenol using the solvent evaporation method at a small scale $(100 \mathrm{~mL})$. The increase in the production volume up to $2000 \mathrm{~mL}$ did not result in significant changes in particle size, surface charge, encapsulation efficiency and release performance of the formulated microcapsules. Textile substrates were successfully impregnated with the eugenol-loaded CA microspheres through a padding process and the release of this compound throughout time was considered satisfactory.

The developed microspheres as well as the microencapsulation technique, proved to be most suitable for textile functionalization.

Acknowledgments Open access funding provided by Graz University of Technology. This work was developed as part of the Project TexBioPro, POCI-01-0247-FEDER-003472, funded by FEDER, through COMPETE 2020, under PT2020. Patrícia Coimbra acknowledges Portuguese Science Foundation (FCT) for financial support (SFRH-BPD-73367-2010).

Open Access This article is licensed under a Creative Commons Attribution 4.0 International License, which permits use, sharing, adaptation, distribution and reproduction in any medium or format, as long as you give appropriate credit to the original author(s) and the source, provide a link to the Creative Commons licence, and indicate if changes were made. The images or other third party material in this article are included in the article's Creative Commons licence, unless indicated otherwise in a credit line to the material. If material is not included in the article's Creative Commons licence and your intended use is not permitted by statutory regulation or exceeds the permitted use, you will need to obtain permission directly from the copyright holder. To view a copy of this licence, visit http://creativecommons.org/licenses/by/4.0/.

\section{References}

Avérous L, Pollet E (2012) Environmental silicate nano-biocomposites. Green Energy Technol. https://doi.org/10. 1007/978-1-4471-4108-2

Campos E, Branquinho J, Carreira AS et al (2013a) Designing polymeric microparticles for biomedical and industrial applications. Eur Polym J 49:2005-2021. https://doi.org/ 10.1016/j.eurpolymj.2013.04.033

Campos E, Coimbra P, Gil MH (2013b) An improved method for preparing glutaraldehyde cross-linked chitosan-poly(vinyl alcohol) microparticles. Polym Bull 70:549-561. https://doi.org/10.1007/s00289-012-0853-4

Campos E, Branquinho J, Carreira AS et al (2016) Designing polymeric microparticles for biomedical and industrial applications. Eur Polym J 49:2005-2021. https://doi.org/ 10.1016/j.eurpolymj.2013.04.033

Carfagna C, Persico P (2006) Functional textiles based on polymer composites. Macromol Symp 245-246:355-362. https://doi.org/10.1002/masy.200651349

Chandra R, Rustgi R (1998) Biodegradable polymers. Prog Polym Sci 23:1273-1335. https://doi.org/10.1016/S00796700(97)00039-7

Coimbra PA, De Sousa HC, Gil MH (2008) Preparation and characterization of flurbiprofen-loaded poly(3-hydroxybutyrate-co-3-hydroxyvalerate) microspheres. J Microencapsul 25:170-178. https://doi.org/10.1080/ 02652040701814140

Cortés-rojas DF, Souza CRF, Oliveira WP (2014) Encapsulation of eugenol rich clove extract in solid lipid carriers. J Food Eng 127:34-42. https://doi.org/10.1016/j.jfoodeng.2013. 11.027

Doelker E (1993) Cellulose derivatives. Biopolym I Adv Polym Sci 107:199-265. https://doi.org/10.1007/BFb0027554

Fundueanu G, Constantin M, Esposito E et al (2005) Cellulose acetate butyrate microcapsules containing dextran ion-exchange resins as self-propelled drug release system. Biomaterials 26(4337):4337-4347. https://doi.org/10.1016/j. biomaterials.2004.10.036

Ghosh SK (2006) Functional coatings: by polymer microencapsulation. Wiley-VCM, Weinheim

Graham SM, Carlisle R, Choi JJ et al (2014) Inertial cavitation to non-invasively trigger and monitor intratumoral release of drug from intravenously delivered liposomes. J Control Release 178:101-107. https://doi.org/10.1016/j.jconrel. 2013.12.016

Jamekhorshid A, Sadrameli SM, Farid M (2014) A review of microencapsulation methods of phase change materials (PCMs) as a thermal energy storage (TES) medium. Renew Sustain Energy Rev 31:531-542. https://doi.org/10.1016/j. rser.2013.12.033 
Jelvehgari M, Montazam SH (2012) Comparison of microencapsulation by emulsion-solvent extraction/evaporation technique using derivatives cellulose and acrylatemethacrylate copolymer as carriers. Jundishapur J Nat Pharm Prod 7:144-152. https://doi.org/10.5812/jjnpp.3986

Jyothi NVN, Prasanna PM, Sakarkar SN et al (2010) Microencapsulation techniques, factors influencing encapsulation efficiency. J Microencapsul 27:187-197. https://doi.org/ 10.3109/02652040903131301

Kumar A, Dixit CK (2017) Methods for characterization of nanoparticles. Adv Nanomed Deliv Ther Nucl Acids. https://doi.org/10.1016/B978-0-08-100557-6.00003-1

Kumari A, Yadav SK, Yadav SC (2010) Biodegradable polymeric nanoparticles based drug delivery systems. Colloids Surf B Biointerfaces 75:1-18. https://doi.org/10.1016/j. colsurfb.2009.09.001

Martins IM, Barreiro MF, Coelho M, Rodrigues AE (2014) Microencapsulation of essential oils with biodegradable polymeric carriers for cosmetic applications. Chem Eng J 245:191-200. https://doi.org/10.1016/j.cej.2014.02.024

Miyazaki Y, Ogihara K, Yakou S et al (2003) In vitro and in vivo evaluation of mucoadhesive microspheres consisting of dextran derivatives and cellulose acetate butyrate. Int $\mathbf{J}$ Pharm 258:21-29. https://doi.org/10.1016/S03785173(03)00159-5

Nelson G (2002) Application of microencapsulation in textiles. Int J Pharm 242:55-62

Olaru N, Olaru L, Tudorachi N et al (2013) Nanostructures of cellulose acetate phthalate obtained by electrospinning from 2-methoxyethanol-containing solvent systems: morphological aspects, thermal behavior, and antimicrobial activity. Ind Eng Chem Res 52:696-705. https://doi.org/10. 1021/ie301299d

Pan-in P, Banlunara W, Chaichanawongsaroj N (2014) Ethyl cellulose nanoparticles: clarithomycin encapsulation and eradication of H. pylori. Carbohydr Polym 109:22-27. https://doi.org/10.1016/j.carbpol.2014.03.025

Patil DK, Agrawal DS, Mahire RR, More DH (2016) Synthesis, characterization and controlled release studies of ethyl cellulose microcapsules incorporating essential oil using an emulsion solvent evaporation method. Am J Essent Oils Nat Prod AJEONP 23:23-31

Petrulis D, Petrulyte S (2019) Potential use of microcapsules in manufacture of fibrous products: a review. J Appl Polym Sci 136:1-12. https://doi.org/10.1002/app.47066

Piletti R, Bugiereck AM, Pereira AT et al (2017) Microencapsulation of eugenol molecules by $\beta$-cyclodextrine as a thermal protection method of antibacterial action. Mater
Sci Eng C 75:259-271. https://doi.org/10.1016/j.msec. 2017.02.075

Pramod K, Shahid H, Ansari JA (2013) Development and validation of UV spectrophotometric method for the quantitative estimation of eugenol K. Asian J Pharm Anal 3:43-45. https://doi.org/10.1016/j.phme.2013.08.002

Prasad AK (2007) Novel effects in garment processing and value added finishes. J Text Ass 13:39-42

Rodrigues SN, Martins IM, Fernandes IP et al (2009) Scentfashion ${ }^{\circledR}$ : microencapsulated perfumes for textile application. Chem Eng J 149:463-472. https://doi.org/10.1016/j. cej.2009.02.021

Salaün F (2016) Microencapsulation technology for smart textile coatings. Active coatings for smart textiles. Elsevier, Amsterdam, pp 179-220

Sarier N, Onder E (2007) The manufacture of microencapsulated phase change materials suitable for the design of thermally enhanced fabrics. Thermochim Acta 452:149-160. https://doi.org/10.1016/j.tca.2006.08.002

Shao Y, Wu C, Wu T et al (2018) Eugenol-chitosan nanoemulsions by ultrasound-mediated emulsification: formulation, characterization and antimicrobial activity. Carbohydr Polym 193:144-152. https://doi.org/10.1016/j. carbpol.2018.03.101

Shinde U, Nagarsenker M (2011) Microencapsulation of Eugenol by gelatin-sodium alginate complex coacervation. Indian J Pharm Sci 73:311-315

Sutaphanit P, Chitprasert P (2014) Optimisation of microencapsulation of holy basil essential oil in gelatin by response surface methodology. Food Chem 150:313-320. https:// doi.org/10.1016/j.foodchem.2013.10.159

Torchilin V (2006) Multifunctional nanocarriers $九$. Adv Drug Deliv Rev 58:1532-1555. https://doi.org/10.1016/j.addr. 2006.09.009

Vijayalakshmi Rao R, Ashokan PV, Shridhar MH (2000) Study of cellulose acetate hydrogen phthalate(CAP)-poly methyl methacrylate (PMMA) blends by thermogravimetric analysis. Polym Degrad Stab 70:11-16. https://doi.org/10. 1016/S0141-3910(00)00055-0

Woranuch S, Yoksan R (2013) Eugenol-loaded chitosan nanoparticles: I. Thermal stability improvement of eugenol through encapsulation. Carbohydr Polym 96:578-585. https://doi.org/10.1016/j.carbpol.2012.08.117

Publisher's Note Springer Nature remains neutral with regard to jurisdictional claims in published maps and institutional affiliations. 